\title{
Silencing of LIMD1 promotes proliferation and reverses cell adhesion-mediated drug resistance in non-Hodgkin's lymphoma
}

\author{
JIE TANG ${ }^{1}$, LIQUN ZHU $^{1}$, YUEJIAO HUANG ${ }^{2}$, BING SHI $^{3}$, SHUQING ZHANG $^{3}$, LINGLI GU $^{3}$,

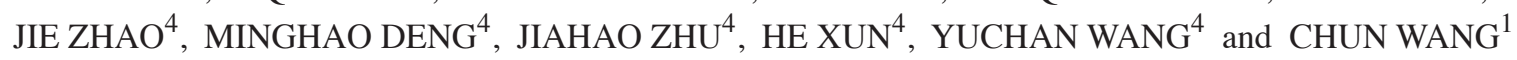 \\ ${ }^{1}$ Department of Oncology, Liyang People's Hospital, Liyang, Jiangsu 213300; \\ ${ }^{2}$ Department of Oncology, Affiliated Cancer Hospital of Nantong University; \\ ${ }^{3}$ Department of Oncology, The Second People's Hospital of Nantong; ${ }^{4}$ Department of \\ Pathogenic Biology, Medical College, Nantong University, Nantong, Jiangsu 226002, P.R. China
}

Received November 20, 2017; Accepted November 8, 2018

DOI: $10.3892 /$ ol.2019.9921

\begin{abstract}
LIM domains-containing protein 1 (LIMD1) is a tumor suppressor protein downregulated in numerous solid malignancies. However, the functional role of LIMD1 in non-Hodgkin's lymphoma (NHL) remains unclear. In the present study, it was demonstrated that LIMD1 is associated with the proliferation of NHL and cell adhesion mediated-drug resistance (CAM-DR). It was indicated by western blot analysis that LIMDlexpression is lower in progressive lymphoma compared with indolent lymphoma. Furthermore, it was indicated that the role of LIMD1 in cell viability and proliferation remains unclear. Finally, the present study demonstrated that LIMD1 serves an important role in CAM-DR by regulating cell cycle progression. Silencing of LIMD1 may reverse CAM-DR in NHL. Therefore, the findings of the present study suggested that LIMD1 may be a potential therapeutic target for patients with NHL.
\end{abstract}

\section{Introduction}

Non-Hodgkin's lymphoma (NHL) is a group of malignant tumors of lymphoid cells, which are classified as indolent or aggressive, according to histopathological and biological characteristics of carcinomatous cells (1-3). The mortality rate of NHL was ranked in the top ten for cancer-associated mortalities worldwide (4). Despite the important

Correspondence to: Professor Yuchan Wang, Department of Pathogenic Biology, Medical College, Nantong University, 19 Qixiu Road, Nantong, Jiangsu 226002, P.R. China

E-mail: wangyuchannt@126.com

Professor Chun Wang, Department of Oncology, Liyang People's Hospital, 70 Jianshe Road, Liyang, Jiangsu 213300, P.R. China E-mail: zlkwangchun@163.com

Key words: non-Hodgkin's lymphoma, LIM domains-containing protein 1 , cell adhesion mediated drug resistance, cell cycle, proliferation improvements in the treatment of NHL over the past decade, to the best of our knowledge, there has been little progress in improving survival for patients with lymphoma (4). Drug resistance remains an obstacle in achieving the optimal treatment of lymphoma (3). Increasing evidence has suggested that the bone marrow microenvironment protects lymphoma cells from apoptosis induced by cytotoxic drugs, including cell adhesion-mediated drug resistance (CAM-DR), leading to relapse following chemotherapy (5-8). The emergence of CAM-DR remains a major obstacle for the successful treatment of lymphoma. However, the underlying molecular mechanisms involved in these processes require further investigation.

LIM domains-containing protein 1 (LIMD1) is a tumor suppressor gene located at chromosome 3 p21.3, a region commonly deleted in carcinomas, including gastric, renal, breast and ovarian cancer (9-13). Loss or downregulation of LIMD1 expression has been reported to lead to abnormal cellular behavior, including perturbation of cell cycle regulation and hyper-proliferation of the cell (14). It has been previously reported that LIMD1 may inhibit S-phase progression in mammalian cells, by downregulating cellular factors necessary for transition from the $G_{0} / G_{1}$ to $S$ phase of the cell cycle (15). In hematologic malignancies, accumulating data suggest that cell cycle arrest may contribute to the CAM-DR phenotype (16-18). Therefore, the present study investigated whether LIMD1 can also affect cell cycle progression and CAM-DR in NHL.

The objective in the present study was to investigate expression and function of LIMD1 in NHL and CAM-DR phenotype. The findings of this study demonstrate for the first time, to the best of our knowledge, that LIMD1 directly affects the proliferation of NHL cells. Furthermore, it was indicated that knockdown of LIMD1 may reverse the CAM-DR phenotype in NHL. Therefore, these findings provide a potential novel therapeutic target for NHL.

\section{Materials and methods}

Patients and specimens. The two cases of pathologically confirmed extra-nodal lymphoma of mucosa-associated 
lymphoid tissues (MALT), follicular lymphoma (FL), mantle cell lymphoma (MCL), diffuse large B cell lymphoma (DLBCL), and reactive lymphoid (RL) were obtained from 4 male and 6 female patients treated at the Second People's Hospital of Nantong (Nantong, China) between April 2016 and February 2017. The age of these patients ranged between 38 and 62 years (mean, 45 years). The collected specimens were subsequently used in western blot analyses. The pathologists were blinded to the study's aim. Written informed consent was obtained from each patient prior to tissue acquisition. Institutional approval was acquired from the Ethical Review Board of The Second People's Hospital of Nantong prior to patient participation in the present study.

Western blot analysis. Western blot analysis was carried out as previously described (19). Tissue extracts were made with a detergent lysis buffer [50 mM Tris ( $\mathrm{pH} 7.4) ; 150 \mathrm{mM} \mathrm{NaCl}$; $1 \%$ NP-40; $0.5 \%$ sodium deoxycholate; $0.1 \%$ SDS; and the protease inhibitor, $1 \mathrm{mmol} / 1$ phenylmethanesulfonyl fluoride]. Equal amounts of protein $(20 \mu \mathrm{g})$ was run on a $10 \%$ SDS-PAGE and transferred to polyvinylidene difluoride filter (PVDF) membranes. The membranes were blocked with 5\% dried skim milk in TBST (20 mM Tris, $150 \mathrm{mM} \mathrm{NaCl,} \mathrm{0.05 \%} \mathrm{Tween-20)}$ at room temperature for $1 \mathrm{~h}$ and subsequently incubated with primary antibody at $4^{\circ} \mathrm{C}$ overnight. Subsequent to washing with TBST at room temperature three times, each for $5 \mathrm{~min}$, the membrane was incubated with HRP-labeled secondary antibody for another $2 \mathrm{~h}$ at room temperature. The membranes were detected with Phototope-HRP western blot detection kit (Cell Signaling Technology, Inc., Danvers, MA, USA), according to the manufacturer's protocols. The antibodies used in this study include: Anti-LIMD1 antibody (dilution, 1:500; cat. no., sc-365050); anti-GAPDH antibody (dilution 1:500; cat. no., sc-25778); anti-proliferating cell nuclear antigen (PCNA) antibody (dilution, 1:1,000; cat. no., sc-56); anti-cyclin E antibody (dilution, 1:500; cat. no., sc-481); anti-cyclin dependent kinase 2 (CDK2) antibody (dilution 1:500; cat. no., sc-53219); and HRP-labeled mouse anti-goat secondary antibody (dilution 1:2,000; cat. no., sc-2354) all purchased from Santa Cruz Biotechnology, Inc. (Dallas, TX, USA).

Cell cultures and stimulation. The DLBCL cell line, OCI-Ly8, and the human mantle cell lymphoma (MCL) cell line Jeko-1 were obtained from Fudan University (Shanghai, China). These cells were all grown in RPMI-1640 medium (Sigma-Aldrich; Merck KGaA, Darmstadt, Germany), supplemented with $10 \%$ fetal bovine serum (FBS; Gibco; Thermo Fisher Scientific, Inc., Waltham, MA, USA). The human bone marrow stromal cell line HS-5, was obtained from the Cell Library, Chinese Academy of Sciences (Shanghai, China) and cultured in F12 medium (Sigma-Aldrich; Merck KGaA) with $10 \%$ FBS. All cell lines were grown at $37^{\circ} \mathrm{C}$ with $5 \% \mathrm{CO}_{2}$.

Cell co-culture. For adhesion assays, lymphoma cell lines OCL-Ly 8 and Jeko-1 $\left(1 \times 10^{6} / \mathrm{ml}\right)$ were adhered to fibronectin-(FN; Sigma-Aldrich; Merck KGaA) and HS-5 cells were plated as described previously (20). Following three washes with PBS to remove unattached cells, adherent cells were used for the subsequent experiments. Lymphoma cells in suspension (SUS) were used as negative control.
Transient transfection. The lymphoma cells lines OCL-Ly8 and Jeko-1 were transfected with $50 \mathrm{nM}$ small interfering RNA (si)-LIMD1 or si-negative control. LIMD1 small interfering (si) RNA was designed and synthesized by GeneChem Co. Ltd., (Shanghai, China). The sequences used were: sense 5'-CTCACT CATGGAGACTATT-3', and antisense 5'-AATAGTCTCCAT GAGTGAGGC-3'. Full-length LIMD1 cDNA were generated using polymerase chain reaction and cloned into pcDNA3.1 construct (Invitrogen; Thermo Fisher Scientific, Inc.), generating the myc-LIMD1 vector. Transfections were performed with the transfection reagent Lipofectamine ${ }^{\circledR} 2000$ (Invitrogen; Thermo Fisher Scientific, Inc.), according to the manufacturer's protocol. Following incubation for $6 \mathrm{~h}$, the medium was replaced with RPMI-1640 containing 10\% FBS. Transfected cells were used for subsequent experiments $48 \mathrm{~h}$ following transfection.

Cell counting assays of viable cells. Lymphoma cells lines OCL-Ly8 and Jeko-1 were seeded onto a 96-well cluster at a density of $1 \times 10^{6} /$ well in a volume of $90 \mu 1$, and then grown overnight with or without the addition of $1 \mu \mathrm{m}$ doxorubicin for $72 \mathrm{~h}$ (Sigma-Aldrich; Merck KGaA). A total of $10 \mu$ l Cell Counting Kit-8 (CCK-8) reagent (Dojindo, Molecular Technologies, Inc., Kumamoto, Japan) was added to the different subset wells, including control-siRNA, LIMD1-siRNA, myc-control and myc-LIMD1 transfected cells and subsequently incubated for an additional $2 \mathrm{~h}$ at $37^{\circ} \mathrm{C}$ with $5 \% \mathrm{CO}_{2}$. The absorbance of the cells was quantitated using a microplate reader (Bio-Rad Laboratories, Inc., Hercules, CA, USA) at a wavelength of $450 \mathrm{~nm}$, with a reference wavelength of $630 \mathrm{~nm}$.

Methylcellulose colony formation assay. Lymphoma cells lines OCL-Ly8 and Jeko-1 were resuspended at $1 \times 10^{3}$ cells/well in semisolid methylcellulose medium (Stem Cell Technologies, Inc., Vancouver, British Columbia, Canada) supplemented with $10 \%$ FBS in 24-well plates. Plates were subsequently maintained for 7 days at $37^{\circ} \mathrm{C}$ with $5 \% \mathrm{CO}_{2}$. Cells were counted using an inverted light microscope (magnification, $\mathrm{x} 5$ ). Data were obtained from three independent experiments.

Flow cytometric analysis. For cell cycle analysis, lymphoma cells lines OCL-Ly8 and Jeko-1 were fixed in 70\% ethanol in PBS at $-20^{\circ} \mathrm{C}$ overnight, subsequently incubated with $1 \mathrm{mg} / \mathrm{ml}$ RNase A at $37^{\circ} \mathrm{C}$ for $30 \mathrm{~min}$ in PBS and then stained with $50 \mu \mathrm{g} / \mathrm{ml}$ propidium iodide at $4^{\circ} \mathrm{C}$ for $30 \mathrm{~min}$ in the dark. Cells were subsequently analyzed using a FACScan ${ }^{\mathrm{TM}}$ flow cytometer (BD Biosciences, Franklin Lakes, NJ, USA).

For cell apoptosis analysis, a flow cytometry assay was performed using an Annexin-V fluorescein isothiocyanate Apoptosis Detection Kit I (BD Biosciences), according to the manufacturer's protocol. Subsequently, the results were quantified using Cell Quest software v.0.9.13 (Becton, Dickinson and Company, Franklin, Lakes, NJ, USA).

Statistical analysis. The data in the present study were analyzed using SPSS v.19.0 software (IBM Corp., Armonk, NY, USA). Data are presented as the mean \pm standard error of the mean. For the comparison of two conditions, the two-tailed t-test was utilized. For testing of multiple groups, the one-way analysis of variance test coupled with the Bonferroni post hoc test was applied. All data shown represent the results of at 

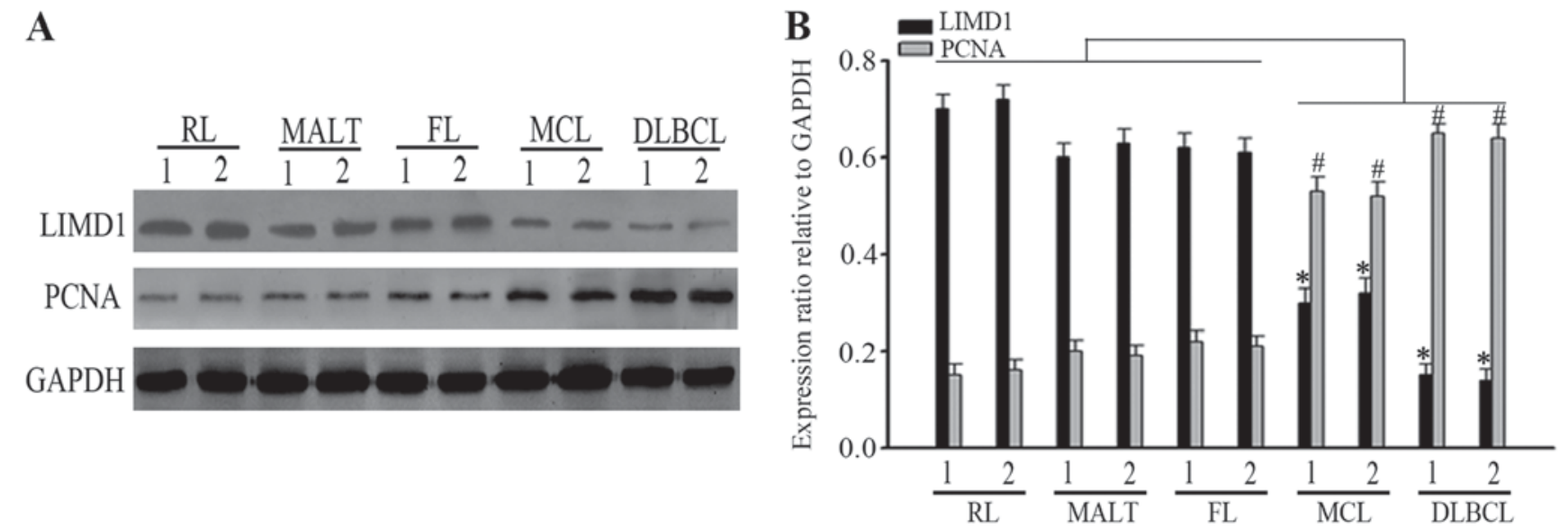

Figure 1. Expression of LIMD1 in clinical specimens. (A) Expression of LIMD1 protein in two cases of RL, MALT, FL, MCL and DLBCL samples. (B) Bar chart of LIMD1 and PCNA protein expression associated with the relative ratio of GAPDH expression. ${ }^{*} \mathrm{P}<0.05 ;{ }^{*} \mathrm{P}<0.05$, compared with the indolent lymphoma (MALT and FL) and RL samples. MALT, mucosa-associated lymphoid tissues; FL, follicular lymphoma; MCL, mantle cell lymphoma; DLBCL, diffuse large B-cell lymphoma; RL, reactive lymphoid; LIMD1, LIM domain-containing protein 1; PCNA, proliferating cell nuclear antigen.

least three independent experiments. $\mathrm{P}<0.05$ was considered to indicate a statistically significant difference.

\section{Results}

Expression of LIMDI in NHL. Previous studies have suggested that LIMD1 is downregulated in numerous solid malignancies, including lung, breast and gastric cancer (10,14,21-23). However, the expression of LIMD1 in NHL remains unclear. To examine the potential role of LIMD1 in NHL, the expression of LIMD1 protein was analyzed by western blotting in two cases of RL, MALT, FL, MCL and DLBCL samples. It was indicated that the expression of LIMD1 was downregulated in progressive lymphoma, MCL and DLBCL, compared with in indolent lymphoma, MALT and FL, and RL samples (Fig. 1A). Furthermore, it was indicated that the expression of PCNA, a proliferative marker, was inversely associated with LIMD1 expression (Fig. 1B).

LIMD1 affects proliferation in NHL. The present study demonstrated that LIMD1 was inversely associated with PCNA expression. Therefore, it was speculated that LIMD1 may serve a role in regulating the proliferation of NHL cells. To validate this hypothesis, the NHL cell lines OCI-Ly8 and Jeko-1 were used to examine the potential effect of LIMD1 on proliferation. To investigate the effect of LIMD1 on cell proliferation, transfection of LIMD1 in NHL cell lines was performed and transfection efficiency was confirmed by western blot analysis (Fig. 2A). Subsequently, CCK-8 assays and Methylcellulose colony formation assays were used to measure the effect of LIMD1 on cell growth. As expected, the knockdown of LIMD1 resulted in a significant increase in proliferation rate (Fig. 2B and C) and colony formation ability (Fig. 2D-G) in OCI-Ly8 and Jeko-1 cells compared with the control group $(\mathrm{P}<0.05)$. By contrast, overexpression of LIMD1 resulted in a significant inhibition of OCI-Ly8 and Jeko-1 cell proliferation rate (Fig. $2 \mathrm{~B}-\mathrm{G})(\mathrm{P}<0.05$, compared with the control group). Therefore, the aforementioned results support the anti-proliferative role of LIMD1 in NHL cells.
LIMD1 inhibits cell cycle progression. Previous studies have indicated that LIMD1 serves a crucial anti-proliferation role by downregulating cellular factors, including PCNA and cyclin E, which transit cells from the $\mathrm{G}_{0} / \mathrm{G}_{1}$ to the $S$ phase of the cell cycle $(14,15)$. Therefore, the expression of PCNA and cyclin E was evaluated in OCI-Ly8 and Jeko-1 cells. It was indicated that knockdown of LIMD1 resulted in a significant increase $(\mathrm{P}<0.05)$ of cyclin $\mathrm{E}, \mathrm{CDK} 2$ and PCNA expression compared with that in control-siRNA transfected cells (Fig. 3A). By contrast, overexpression of LIMD1 resulted in a significant decrease ( $\mathrm{P}<0.05$, compared with the control group) in the expression of the aforementioned proteins (Fig. 3A). In addition, flow cytometric analysis indicated that the knockdown of LIMD1 in NHL cells accelerated transit from the $\mathrm{G}_{0} / \mathrm{G}_{1}$ phase to the $\mathrm{S}$ phase (Fig. 3B and C). Conversely, overexpression of LIMD1 led to a significant increase $(\mathrm{P}<0.05$, compared with the control group) in the percentage of NHL cells arrested in the $G_{0} / G_{1}$ phase (Fig. 3B and C). Therefore, these results indicate that LIMD1 may inhibit $\mathrm{G}_{0} / \mathrm{G}_{1}$ to $\mathrm{S}$ phase transition by inducing $\mathrm{G}_{0} / \mathrm{G}_{1}$ arrest.

Knockdown of LIMD1 reverses the CAM-DR phenotype. Increasing evidence indicates that the adhesion of lymphoma cells to extracellular matrices or stromal cells in the bone marrow microenvironment induces cell cycle arrest and protects cells from drug-induced apoptosis $(16,24,25)$. Based on the results, the present study proposed that the anti-proliferation role of LIMD1 should be one of the major reasons for CAM-DR in NHL cells. To investigate the role of LIMD1 in the CAM-DR phenotype, cell adhesion assays were used to examine the expression of LIMD1. It was indicated that adhesion of NHL cells to FN and HS-5 cells significantly increased $(\mathrm{P}<0.05)$ LIMD1 expression, compared with their suspension (SUS) counterparts (Fig. 4A and B). Subsequent cell viability assays revealed that adhesion to FN and HS-5 cells significantly protected $(\mathrm{P}<0.05)$ NHL cells from doxorubicin-induced cytotoxicity compared with the same cell types cultured in SUS, as these effects were attenuated by the downregulation of LIMD1 (Fig. 4C). Flow cytometry assays verified an 
A

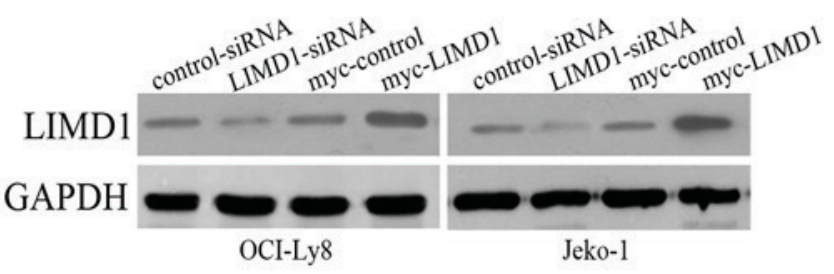

B

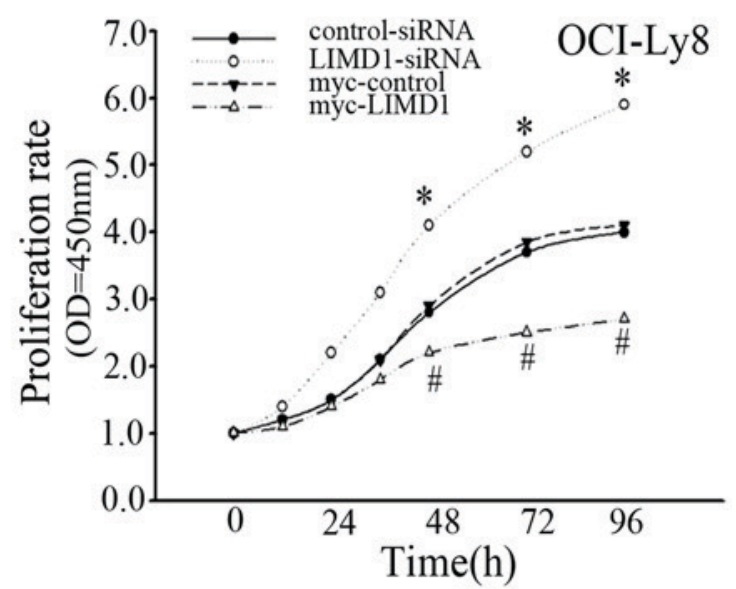

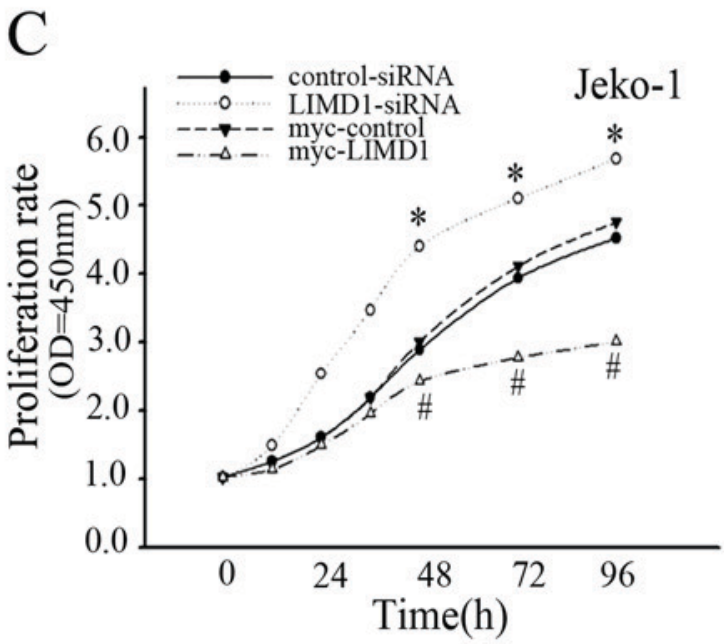

$\mathrm{F}$

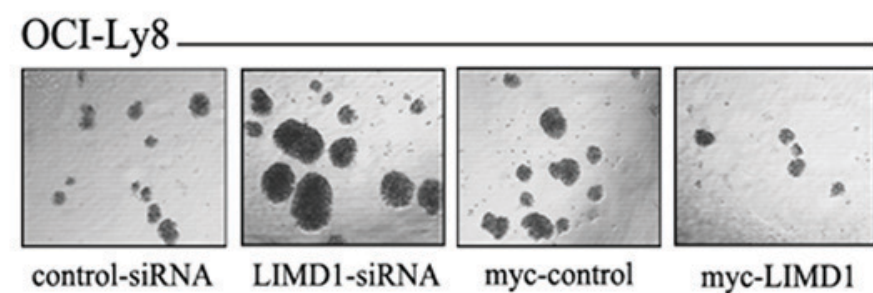

control-siRNA LIMD1-siRNA myc-control
myc-LIMD1

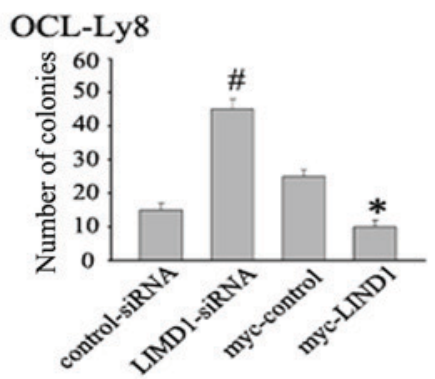

$\mathrm{E}$

Jeko-1

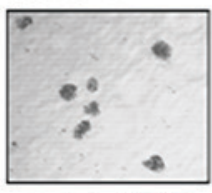

control-siRNA

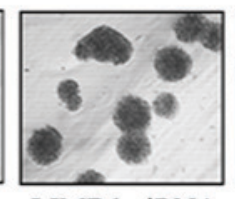

LIMD1-siRNA

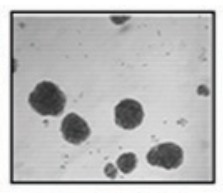

myc-control

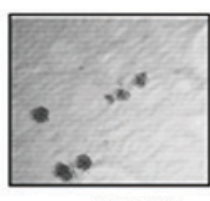

myc-LIMD1
G

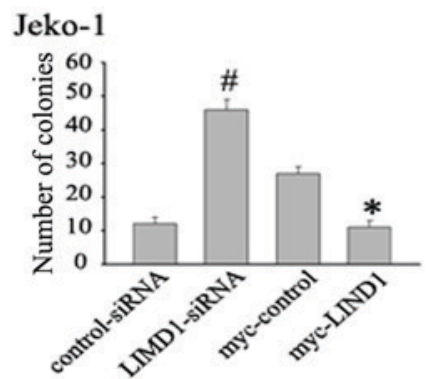

Figure 2. LIMD1 affects proliferation in NHL. (A) Transfection of OCI-Ly8 cells and Jeko-1 cells with either LIMD1-siRNA or myc-LIMD1, or their respective controls. Their interference efficiencies were elevated by western blotting as the expression of LIMD1 was decreased by treatment of LIMD1-siRNA and the expression of LIMD1 was increased by treatment of myc-LIMD1.CK-8 assays to evaluate proliferation rate at the indicated time in (B) OCI-Ly8 cells and $(\mathrm{C})$ Jeko-1 cells transfected with either LIMD1-siRNA or myc-LIMD1 or their respective controls. ${ }^{~} \mathrm{P}<0.05$ compared with control-siRNA; ${ }^{\prime \prime} \mathrm{P}<0.05$ compared with Myc-control. Representative images of colonies from LIMD1-siRNA or myc-LIMD1 transfected (D) OCI-Ly8 cells and (E) Jeko-1 cells (magnification, 5x). Quantitative analysis of colony formation ability in (F) OCI-Ly8 cells and (G) Jeko-1 cells. ${ }^{*} \mathrm{P}<0.05$ compared with control-siRNA; ${ }^{*} \mathrm{P}<0.05$ compared with Myc-control. LIMD1, LIM domain-containing protein 1; si, small interfering; OD, optical density.

increase in drug-induced apoptotic cells among LIMD1-siRNA transfected cells compared with the controls (Fig. 4D). Taking into consideration all aforementioned findings, the present study data indicate that knockdown of LIMD1 expression may reverse the CAM-DR phenotype in NHL.

\section{Discussion}

NHL is a group of malignant neoplasms in the lymph tissues, which includes DLBCL, MCL, FL and MALT $(3,7)$. Although standard systemic chemotherapy remains the main therapy 

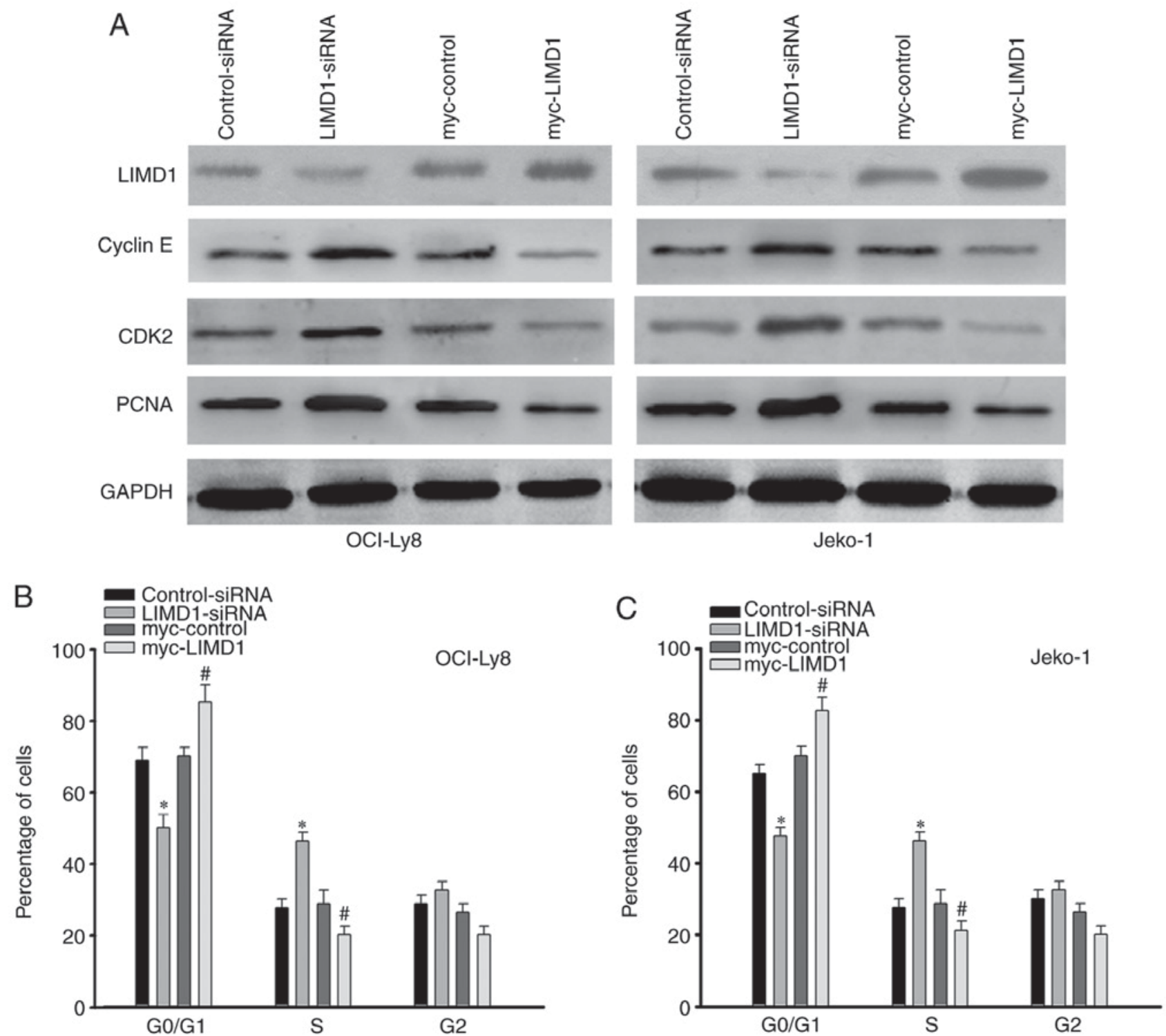

Figure 3. LIMD1 inhibits cell cycle progression. (A) OCI-Ly8 cells and Jeko-1 cells were transfected with either LIMD1-siRNA or myc-LIMD1 or their respective controls and analyzed for LIMD1, cyclin E, CDK2 and PCNA expression. (B) OCI-Ly8 cells and (C) Jeko-1 cells were transfected with either LIMD1-siRNA or Myc-LIMD1 or their respective controls to examine transitions from the $\mathrm{G}_{0} / \mathrm{G}_{1}$ to $\mathrm{S}$ phases. ${ }^{*} \mathrm{P}<0.05$ compared with control-siRNA; ${ }^{*} \mathrm{P}<0.05$ compared with Myc-control. LIMD1, LIM domain-containing protein 1; si, small interfering; PCNA, proliferating cell nuclear antigen; CDK2, anti-cyclin dependent kinase 2 .

option, the emergence of clinical drug resistance usually leads to therapy failure $(4,25)$. It has been documented that the bone marrow microenvironment may provide components necessary for cell survival, growth and the development of acquired multidrug resistance (26-29). This environment consists of hematopoietic cells, stromal cells and the extracellular matrix. It also indicates that the tumor cells that adhered to components of the bone marrow microenvironment may block apoptosis induced by chemotherapy agents, including CAM-DR $(5,16,17)$. The emergence of multidrug resistance remains a major obstacle for the successful treatment of NHL.

Previous studies have demonstrated that adhesion of hematopoietic tumors to FN may block cell cycle progression, and that this is associated with drug resistance $(16,17)$. Furthermore, cell cycle arrest has been reported to be associated with the inhibition of CDK2 $(3,4,25)$. It has been reported that LIMD1 may prevent the entry of cells into the
S phase by downregulating genes involved in $\mathrm{G} 1$ to $\mathrm{S}$ phase transitions $(14,15)$. Based on these findings, the expression of LIMD1 in NHL cells and the effect of LIMD1 on CAM-DR in OCI-Ly8 and Jeko-1 NHL cell lines were investigated. In the present study, it was indicated that LIMD1 expression was downregulated in progressive lymphomas, namely MCL and DLBCL, compared with in indolent lymphomas, namely MALT and FL tissues. Subsequently, it was indicated that LIMD1 inhibited proliferation by inducing cell cycle arrest and suppressing genes involved in the G1/S transition. In addition, it was indicated that LIMD1 participated in the process of CAM-DR in NHL. In the present study it was demonstrated that adhesion of OCI-Ly8 and Jeko-1 cells to FN or HS-5 cells significantly increased LIMD1 expression compared with cells cultured in SUS. It was subsequently verified that adhesion to FN or HS-5 cells may protect OCI-Ly8 and Jeko-1 cells from doxorubicin-induced cytotoxicity compared with their SUS 
A

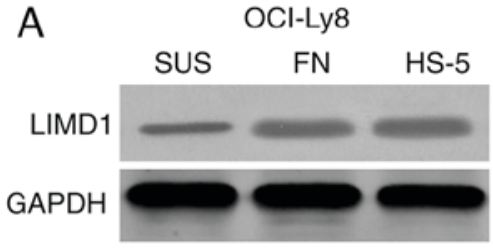

B
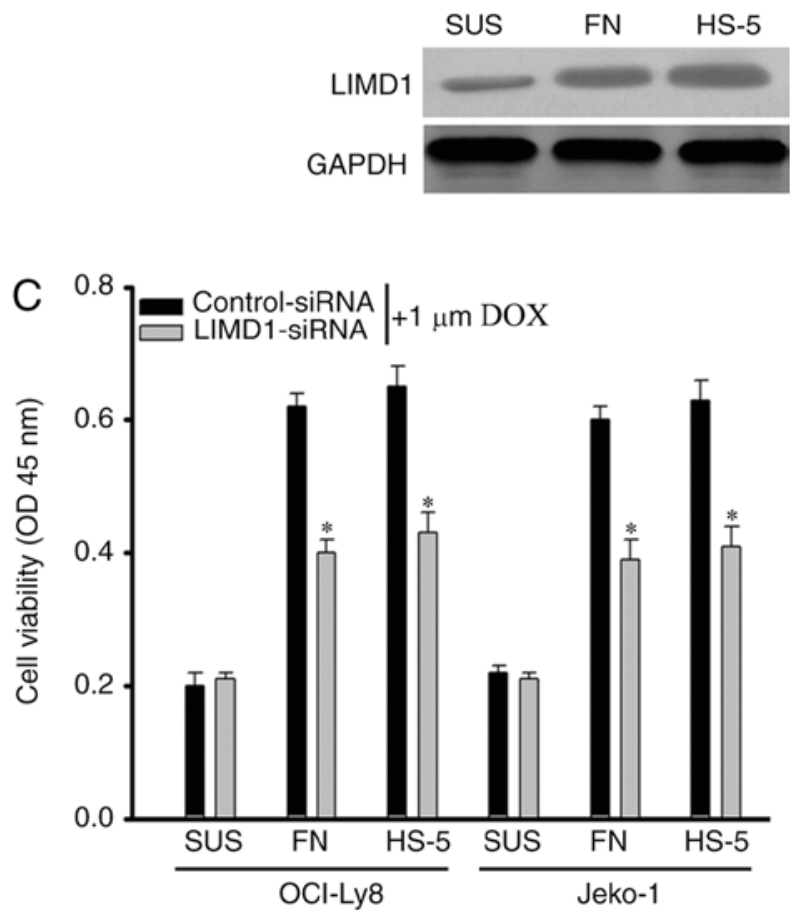
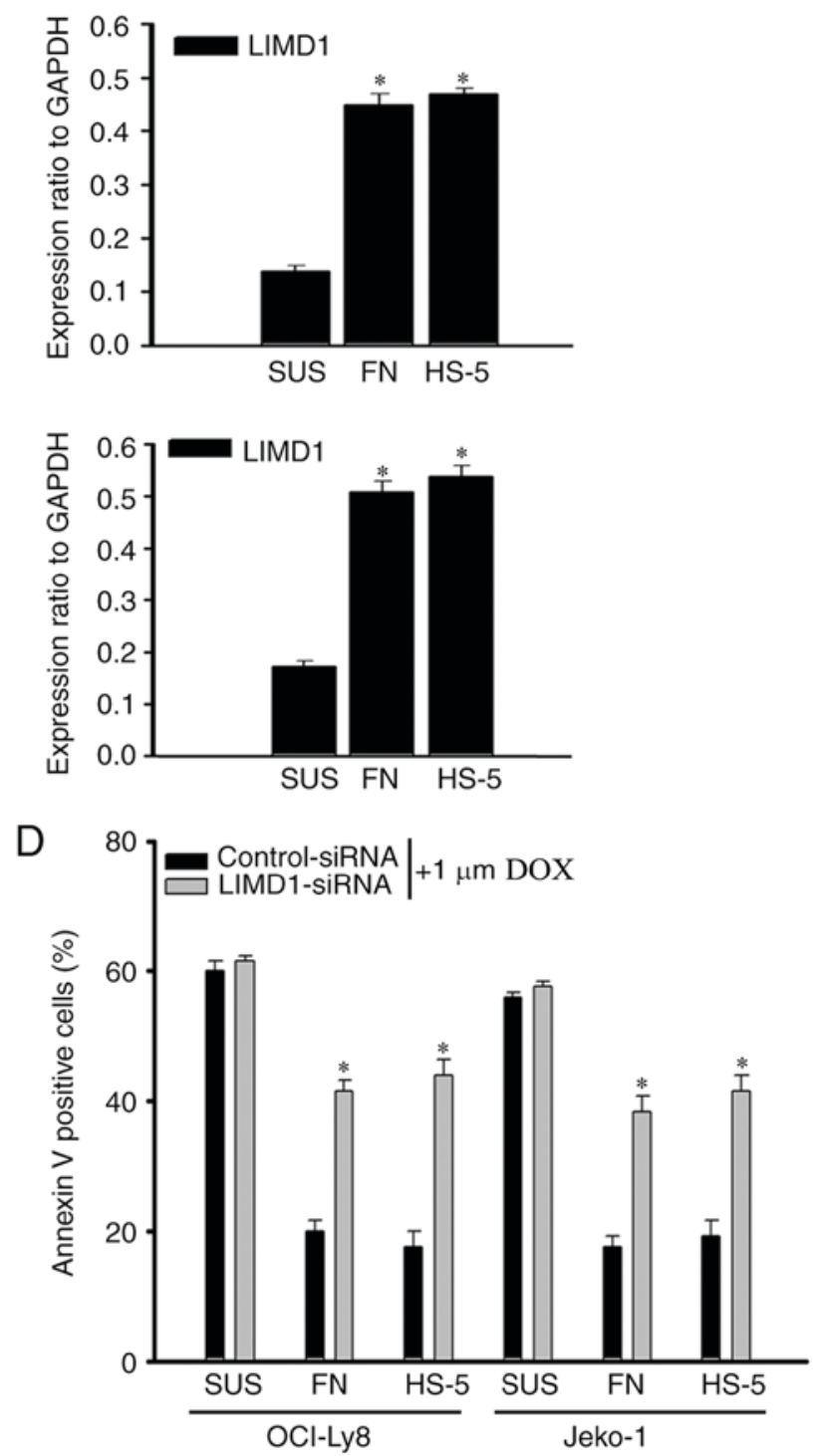

Figure 4. Knockdown of LIMD1 reverses the CAM-DR phenotype. (A) OCI-Ly8 and (B) Jeko-1 cells were adhered to FN or HS-5 cells, or cultured SUS, to detect the expression of LIMD1. The bar chart demonstrates the LIMD1 protein expression ratio relative to GAPDH. *P<0.05, compared with cells in SUS. (C) Viability of OCI-Ly8 and Jeko-1 cells transfected with control-siRNA or LIMD1-siRNA and adhered to FN, HS-5 cells, or cultured in SUS along with DOX treatment. (D) DOX-induced cell apoptosis in OCI-Ly8 and Jeko-1 cells transfected with control-siRNA or LIMD1-siRNA adhered to FN, HS-5 cells or cultured SUS along with DOX treatment. ${ }^{*} \mathrm{P}<0.05$, compared with cells in SUS. CAM-DR, cell adhesion-mediated drug resistance; SUS, suspension; si, small interfering; LIMD1, LIM domain-containing protein 1; FN, fibronectin; DOX, doxorubicin; OD, optical density.

counterparts, while the protective effect was reversed when the cells were transfected with LIMD1-siRNA.

LIMD1, a member of the ZYXIN family of proteins, is widely expressed in human and mouse tissues $(30,31)$. Previous research has demonstrated that LIMD1 directly interacts with retinoblastoma and inhibits E2F transcription factor 1-mediated transcription $(13,23)$. Furthermore, it has been reported that upregulation of LIMD1 inhibited tumor growth in $80 \%$ of lung cancers, breast cancers and head squamous cell carcinomas, indicating possible tumor-suppressing functions (30). LIMD1 is a tumor suppressor gene encoded at chromosome 3 p21.3, a region usually lost in a number of solid tumors, including lung, breast, gastric, ovarian and renal cancer $(9-12,21)$. It has been reported that loss or downregulation of LIMD1 expression may led to a cancerous phenotype, due to its anti-proliferative properties (15).
However, the function of LIMD1 in the development of NHL remains unclear. In the present study, it was demonstrated that LIMD1 may inhibit the proliferation of NHL cells by inducing cell cycle arrest, and that knockdown of LIMD1 may reverse the CAM-DR phenotype in NHL cells.

In summary, the present study demonstrated for the first time, to the best of our knowledge, that LIMD1 may block cell cycle progression and downregulate genes involved in the G1 to $S$ phase transition in NHL. Silencing of LIMD1 could reverse the CAM-DR phenotype in NHL. Therefore, based on the data, it is proposed that the inhibition of LIMD1 expression may be a novel effective strategy for the treatment of NHL.

\section{Acknowledgements}

Not applicable. 


\section{Funding}

The present study was supported by the Key Research Project of Liyang Science and technology Bureau (grant no. LC 2016009), the National Natural Science Foundation of China (grant no. 81600158), the Six Talent Peaks Project in Jiangsu Province (grant no. 2015-SWYY-021), the Natural Science Foundation of Jiangsu province Grants (grant nos. BK2012231, BK20160060), the Major Research of Natural Science Foundation for Colleges and Universities in Jiangsu Province (grant no. 16KJ320004) and the Innovative Training Program for Undergraduates in Nantong University and Nantong Science and Technology Projects (grant nos. QYZ15069, MS22016059 and YYZ16026).

\section{Availability of data and materials}

All data generated or analyzed during this study are included in this published article.

\section{Authors' contributions}

YH, BS, SZ, LG, JZ, MD, JHZ and HX were responsible for the completion of experiments. JT, LZ, YW and CW undertook study design and wrote the manuscript All authors have read and approved the final version of the manuscript.

\section{Ethics approval and consent to participate}

Written informed consent was obtained from each patient prior to tissue acquisition. Institutional approval was acquired from the Ethical Review Board of The Second People's Hospital of Nantong prior to patient participation in the present study.

\section{Patient consent for publication}

All the patients provided written informed consent for the publication of any associated data and accompanying images.

\section{Competing interests}

All the authors declare no competing interests.

\section{References}

1. Gayle S, Landrette S, Beeharry N, Conrad C, Hernandez M, Beckett P, Ferguson SM, Mandelkern T, Zheng M, Xu T, et al: Identification of apilimod as a first-in-class PIKfyve kinase inhibitor for treatment of B-cell non-hodgkin lymphoma. Blood 129: 1768-1778, 2017.

2. Riley SN: Investigating the multivariate nature of NHL player performance with structural equation modeling. PLoS one 12: e0184346, 2017.

3. Yin H, Miao X, Wu Y, Wei Y, Zong G, Yang S, Chen X, Zheng G, Zhu X, Guo Y, et al: The role of the chaperonin containing t-complex polypeptide 1 , subunit 8 (CCT8) in B-cell non-hodgkin's lymphoma. Leuk Res 45: 59-67, 2016.

4. Zhu X, Miao X, Wu Y, Li C, Guo Y, Liu Y, Chen Y, Lu X, Wang Y and He S: ENO1 promotes tumor proliferation and cell adhesion mediated drug resistance (CAM-DR) in non-hodgkin's lymphomas. Exp Cell Res 335: 216-223, 2015.

5. Hazlehurst LA and Dalton WS: Mechanisms associated with cell adhesion mediated drug resistance (CAM-DR) in hematopoietic malignancies. Cancer Metastasis Rev 20: 43-50, 2001 .
6. Landowski TH, Olashaw NE, Agrawal D and Dalton WS: Cell adhesion-mediated drug resistance (CAM-DR) is associated with activation of NF-kappa B (RelB/p50) in myeloma cells. Oncogene 22: 2417-2421, 2003.

7. Miao X, Xu X, Wu Y, Zhu X, Chen X, Li C, Lu X, Chen Y, Liu Y, Huang J, et al: Overexpression of TRIP6 promotes tumor proliferation and reverses cell adhesion-mediated drug resistance (CAM-DR) via regulating nuclear p27(Kip1) expression in non-hodgkin's lymphoma. Tumour Biol 37: 1369-1378, 2016.

8. Nakagawa Y, Nakayama H, Nagata M, Yoshida R, Kawahara K, Hirosue A, Tanaka T, Yuno A, Matsuoka Y, Kojima T, et al: Overexpression of fibronectin confers cell adhesion-mediated drug resistance (CAM-DR) against 5-FU in oral squamous cell carcinoma cells. Int J Oncol 44: 1376-1384, 2014.

9. Hou X, Li T, Ren Z and Liu Y: Novel BRCA2-interacting protein, LIMD1, is essential for the centrosome localization of BRCA2 in esophageal cancer cell. Oncol Res 24: 247-253, 2016.

10. Sharp TV, Al-Attar A, Foxler DE, Ding L, de A Vallim TQ, Zhang Y, Nijmeh HS, Webb TM, Nicholson AG, Zhang Q, et al: The chromosome 3p21.3-encoded gene, LIMD1, is a critical tumor suppressor involved in human lung cancer development. Proc Natl Acad Sci USA 105: 19932-19937, 2008.

11. Spendlove I, Al-Attar A, Watherstone O, Webb TM, Ellis IO, Longmore GD and Sharp TV: Differential subcellular localisation of the tumour suppressor protein LIMD1 in breast cancer correlates with patient survival. Int J Cancer 123: 2247-2253, 2008.

12. Huggins CJ, Gill M and Andrulis IL: Identification of rare variants in the hLIMD1 gene in breast cancer. Cancer Genet Cytogenet 178: 36-41, 2007.

13. Kiss H, Kedra D, Yang Y, Kost-Alimova M, Kiss C, O'Brien KP, Fransson I, Klein G, Imreh S and Dumanski JP: A novel gene containing LIM domains (LIMD1) is located within the common eliminated region 1 (C3CER1) in 3p21.3. Hum Genet 105: 552-559, 1999.

14. Huggins CJ and Andrulis IL: Cell cycle regulated phosphorylation of LIMD1 in cell lines and expression in human breast cancers. Cancer Lett 267: 55-66, 2008.

15. Mayank AK, Sharma S, Deshwal RK and Lal SK: LIMD1 antagonizes E2F1 activity and cell cycle progression by enhancing Rb function in cancer cells. Cell Biol Int 38: 809-817, 2014.

16. Hang Q, Fei M, Hou S, Ni Q, Lu C, Zhang G, Gong P, Guan C, Huang $X$ and $\mathrm{He}$ S: Expression of Spy1 protein in human non-Hodgkin's lymphomas is correlated with phosphorylation of p27 Kip1 on Thr187 and cell proliferation. Med Oncol 29: 3504-3514, 2012.

17. Hazlehurst LA, Damiano JS, Buyuksal I, Pledger WJ and Dalton WS: Adhesion to fibronectin via betal integrins regulates p27kipl levels and contributes to cell adhesion mediated drug resistance (CAM-DR). Oncogene 19: 4319-4327, 2000.

18. Sarkar S, Maiti GP, Jha J, Biswas J, Roy A, Roychoudhury S, Sharp T and Panda CK: Reduction of proliferation and induction of apoptosis are associated with shrinkage of head and neck squamous cell carcinoma due to neoadjuvant chemotherapy. Asian Pac J Cancer Prev 14: 6419-6425, 2013.

19. Huang X, Wang Y, Nan X, He S, Xu X, Zhu X, Tang J, Yang X, Yao L, Wang $X$ and Cheng $C$ : The role of the orphan $G$ protein-coupled receptor 37 (GPR37) in multiple myeloma cells. Leuk Res 38: 225-235, 2014.

20. Damiano JS, Cress AE, Hazlehurst LA, Shtil AA and Dalton WS: Cell adhesion mediated drug resistance (CAM-DR): Role of integrins and resistance to apoptosis in human myeloma cell lines. Blood 93: 1658-1667, 1999.

21. Chen Z, Zhu X, Xie T, Xie J, Quo K and Liu X: Drug resistance reversed by silencing LIM domain-containing protein 1 expression in colorectal carcinoma. Oncol Lett 8: 795-798, 2014.

22. Ghosh S, Ghosh A, Maiti GP, Alam N, Roy A, Roy B, Roychoudhury S and Panda CK: Alterations of $3 \mathrm{p} 21.31$ tumor suppressor genes in head and neck squamous cell carcinoma: Correlation with progression and prognosis. Int J Cancer 123: 2594-2604, 2008.

23. Ghosh S, Ghosh A, Maiti GP, Mukherjee N, Dutta S, Roy A, Roychoudhury $\mathrm{S}$ and Panda CK: LIMD1 is more frequently altered than RB1 in head and neck squamous cell carcinoma: Clinical and prognostic implications. Mol Cancer 9: 58, 2010.

24. Tang J, Ji L, Wang Y, Huang Y, Yin H, He Y, Liu J, Miao X, Wu Y, Xu X, et al: Cell adhesion down-regulates the expression of vacuolar protein sorting 4B (VPS4B) and contributes to drug resistance in multiple myeloma cells. Int J Hematol 102: 25-34, 2015. 
25. Ouyang Y, Zhong F, Wang Q, Ding L, Zhang P, Chen L, Wang Y and Cheng C: DIXDC1 promotes tumor proliferation and cell adhesion mediated drug resistance (CAM-DR) via enhancing p-Akt in Non-Hodgkin's lymphomas. Leuk Res 50: 104-111, 2016.

26. Balsas P, Palomero J, Eguileor Á, Rodríguez ML, Vegliante MC, Planas-Rigol E, Sureda-Gómez M, Cid MC, Campo E and Amador V: SOX11 promotes tumor protective microenvironment interactions through CXCR4 and FAK regulation in mantle cell lymphoma. Blood 130: 501-513, 2017.

27. Bjorklund CC, Baladandayuthapani V, Lin HY, Jones RJ, Kuiatse I, Wang H, Yang J, Shah JJ, Thomas SK, Wang M, et al: Evidence of a role for CD44 and cell adhesion in mediating resistance to lenalidomide in multiple myeloma: Therapeutic implications. Leukemia 28: 373-383, 2014.
28. Tsubaki M, Takeda T, Yoshizumi M, Ueda E, Itoh T, Imano M, Satou T and Nishida S: RANK-RANKL interactions are involved in cell adhesion-mediated drug resistance in multiple myeloma cell lines. Tumour Biol 37: 9099-9110, 2016.

29. Waldschmidt JM, Simon A, Wider D, Müller SJ, Follo M, Ihorst G, Decker S, Lorenz J, Chatterjee M, Azab AK, et al: CXCL12 and CXCR7 are relevant targets to reverse cell adhesion-mediated drug resistance in multiple myeloma. Br J Haematol 179: 36-49, 2017.

30. Foxler DE, James V, Shelton SJ, Vallim TQ, Shaw PE and Sharp TV: PU.1 is a major transcriptional activator of the tumour suppressor gene LIMD1. FEBS Lett 585: 1089-1096, 2011.

31. Zhao MK, Wang Y, Murphy K, Yi J, Beckerle MC and Gilmore TD: LIM domain-containing protein trip6 can act as a coactivator for the v-Rel transcription factor. Gene Exp 8: 207-217, 1999. 